\title{
Analysis of Results in Simulation and Modeling of CDMA Systems
}

\author{
Samad S. Kolahi \\ Unitec New Zealand \\ skolahi@unitec.ac.nz
}

\begin{abstract}
In this paper, using discrete event stochastic simulation by batch-means, new results have been obtained by analysing the sensitivity of CDMA blocking probability for a given traffic load against various number of calls per batch and confidence intervals. It is found that for the system under study one long simulation with one million call arrivals produce approximately 99\% confidence in results while it needs 100,000 calls to achieve 95\% confidence. For system under study and with 27 Erlang of traffic, the blocking probability is 0.0202 with $99 \%$ confidence and 0.0192 with 95\% confidence. The impact of warm-up period on CDMA simulation is discussed. Situation with three tiers of neighbouring cells are considered when mobile compares three base stations and chooses the base station with the strongest signal.
\end{abstract}

\section{Introduction}

The operation of communication networks is usually evaluated using simulation techniques which, unlike analytical methods, use fewer assumptions and behave more like real systems. The complexity of modern communication systems is another driving force behind widespread use of simulation techniques $[1,2]$. Performance modeling by simulation generally includes the following steps: (a) obtain a valid simulation model of the system; (b) develop, test, and verify simulation software; (c) obtain and analyze data [3].

Because in stochastic simulation the input data (for example arrival and service times) are random variables, the output results might vary depending on the simulation length. Statistical behavior and analysis of the random variables is very important in determining the accuracy of the simulation results [3]. In addition, the simulation model is an abstract model and the results are not as deterministic as the hardware model's results. Therefore, the CDMA simulation model will need to be run many times, with different random numbers, to provide sample results for analysis [4]. In this paper, discrete event simulation by batchmeans of a $\mathrm{M} / \mathrm{M} / \infty$ queuing system is utilized to analyze the simulation results. The simulation warmup period, run time, and CDMA blocking probability results are analyzed by varying the number of calls per batches and the confidence interval in the batch-means simulation method. The warm-up period is also studied because initially the queuing system is empty and it takes some time for the system to reach the steady-state situation. Call blockage is less unlikely to occur during this period. Any data collected during the initial period (the transient or warm-up period) could affect the accuracy of the results.

The organization of this paper is as follows. In next section, the system model is briefly discussed. Section 3 covers simulation by batch-means. The simulation analysis results are then presented. The last section presents conclusions.

\section{System Model}

A cellular CDMA system (a home cell and three tiers of neighboring cells as discussed in [5] ) is considered with a base station located at the centre of each cell. The signal strengths of three neighboring cells are compared to find the home cell as found in [6]. Calls are considered to suffer log-normal shadowing (but not Rayleigh fading [7]). All cells are assumed to be homogeneous in every respect and users are assumed to be uniformly distributed over the cell area. The reverse link (mobile to base station) is investigated as it is the limiting link due to its inferior performance compared to the forward link $[8,9]$. The simulation model and blocking probability calculations are further explained in $[6,10,11]$. 


\section{Simulation by batch-means method}

A variety of methods (replications method, batch means method, and regenerative simulations) have been suggested for data collection and analysis [12]. The batch-means method is used in this study to simulate the CDMA system. In this method, the results are obtained from a single simulation run time but the simulation observations are divided into batches [13]. The simulation is run for a number of batches and continued until the required precision is reached between the results of various simulation batches [12].

The observations from $K$ batches with $n$ observation per batch are

$$
\begin{aligned}
& \left(X_{11}, X_{12}, X_{13} \ldots \ldots \ldots \ldots . . . X_{1 n}\right),\left(X_{21}, X_{22}, X_{23} \ldots \ldots \ldots \ldots . . . X_{2 n}\right), \\
& \ldots .,\left(X_{K 1}, X_{K 2}, X_{K 3} \ldots \ldots \ldots \ldots . . . X_{K n}\right)
\end{aligned}
$$

As the first batch has a warm-up period, and assuming $w$ observations belong to initial warm-up period, the mean value of results for the first batch $\left(\mu_{l}\right)$ is [12]

$$
\mu_{1}=\frac{1}{n-w} \sum_{j=1}^{n} X_{1 j}
$$

The mean value of results per batch, $\left(\mu_{2}\right), \ldots \ldots$ ,$\left(\mu_{n}\right)$ can be calculated as

$$
\mu_{i}=\frac{1}{n} \sum_{j=2}^{n} X_{i j}
$$

and the sample mean of the various iterations is

$$
\bar{\mu}=\frac{1}{K} \sum_{i=1}^{K} \mu_{i}
$$

so that $\bar{\mu}$ is the average of the averages. The sample variance for the iterations is thus

$$
\sigma^{2}(K)=\frac{1}{K-1} \sum_{i=1}^{K}\left[\mu_{i}-\bar{\mu}\right]^{2}
$$

Applying the Central Limit Theorem[14-16], for a very large number of call arrivals, it is fair to assume a normal distribution for $\mu_{i}$ with an estimated mean $\bar{\mu}$ and standard deviation $\sigma(\mathrm{k}) / \sqrt{K}$. For a normally distributed variable, the $95 \%$ confidence interval is \pm 1.96 times the standard deviation of the mean and the
99\% confidence interval is \pm 2.58 times the standard deviation of the mean [15]. If $\overline{\bar{\mu}}$ is the true mean, then the $99 \%$ confidence interval is

$$
P\left(-2.58 \frac{\sigma}{\sqrt{K}}<\overline{\bar{\mu}}-\bar{\mu}<+2.58 \frac{\sigma}{\sqrt{K}}\right)=0.99
$$

Hence the true mean $\overline{\bar{\mu}}$ is $\bar{\mu} \pm 2.58 \sigma(K) / \sqrt{K}$ with 99\% confidence and $\bar{\mu} \pm 1.96 \sigma(K) / \sqrt{K}$ with $95 \%$ confidence [17]. The difference $\overline{\bar{\mu}}-\bar{\mu}$ is the error of the estimate, the difference between the true mean and the estimated mean. If the results within a $1 \%$ margin are accepted, the following inequity should hold to terminate the simulation

$$
\frac{2.58 \sigma(K) / \sqrt{K}}{\bar{\mu}}<0.01
$$

Note that as $K$ (number of batches) increases, the LHS of the above inequality decreases. Essentially (7) is a test, the batches are continued until it is satisfied. In the CDMA simulation model, and for each batch, the mean value of the average blocking probability is obtained in Section 5 for 5,000 and 50,000 call arrivals. When the number of call arrivals exceeds 5000 or 50,000 , the second batch begins. The batches are continued until the required $95 \%$ or $99 \%$ confidence in the results is reached.

\section{Results}

To study the effect of the initial warm-up period on the accuracy of the results, the simulation was performed under various scenarios for the CDMA parameters of $\mathrm{W} / \mathrm{R}=125$ (processing gain), $\alpha=0.4$ (voice activity), $\mathrm{E}_{\mathrm{b}} / \mathrm{N}_{\mathrm{o}}=7 \mathrm{~dB}$ (call quality), $\mathrm{S} / \eta=-1 \mathrm{~dB}$ (signal to background noise), $\sigma_{\xi}=8 \mathrm{~dB}$ (shadowing parameter), and $m=4$ (distance exponent) and traffic load of 27 Erlangs.

Figure 1 shows the effects of the warm-up period when the system has not reached steady state. The simulation was performed with three different sets of random numbers for the first 700 call arrivals. The first few call losses (blocked calls) for the three simulations were call numbers $(62,98,106,113, \ldots)$ and $(79,88,98,202, \ldots)$ and $(124,318,327,379, \ldots)$. Due to randomness of the CDMA parameters, one cannot estimate which calls will be lost. In the three simulations, there were no call losses for the first 61 , 78 , and 123 calls respectively. As it can be seen in Figure 1, initially the system is empty and there is a low probability of call losses. As the system has not 
reached the steady state, there is a large variation in blocking probability and there is not much similarity between the three simulation results. The blocking probability varies between zero and 0.037 . As the simulation progresses and the number of call arrivals increase, variation in the blocking probability drops sharply approaching the $0.015-0.023$ range for 700 call arrivals (Figure 1).

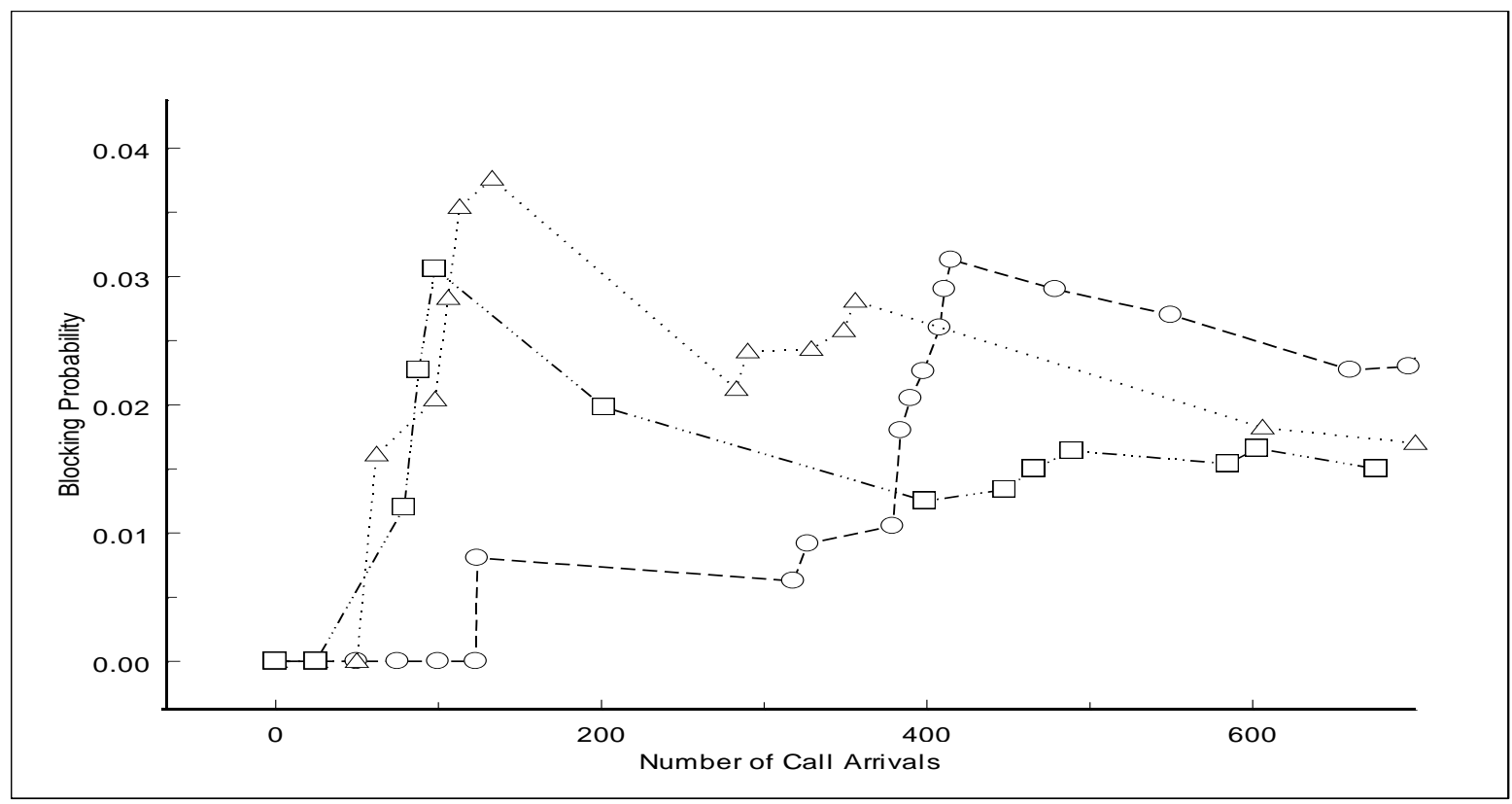

Figure 1: Average blocking probability versus the first 700 calls for three different simulation runs with different random numbers.

The simulation was also performed for two simulation runs with different random numbers, and for the first 10,000 call arrivals, and the blocking probability graph is shown in Figure 2. The warm-up period can be clearly seen in the graph where initially there is a low probability of blockage and data collected during this period should be ignored. As the number of calls increase, the randomness in the results decreases and the results of the two simulation runs approach the blocking probability of 0.018-0.020 (Figure 2).

In the simulation with different number of calls per batch, it was observed that as the number of calls per batch increase, the randomness of the results decreases (Figures 3 and 4). For 5,000 calls per batch, the average blocking probability is within 0.0175-0.021 range and for 50,000 calls per batch it changes within 0.0196-0.0203. The randomness of blocking probabilities in the figures is because of the randomness in the CDMA parameters (call arrivals and departures from the system, shadowing, and call location within the cell). For 5,000 calls per batch,
Figure 3 indicates that the first set of data is not reliable as the blocking probability is low due to warmup period. For the system under study with 27 Erlangs of traffic, if the result within a $95 \%$ confidence interval is required, the batch-mean simulation is performed for twenty batches $(100,000$ total calls) giving the average blockage probability of 0.0192 .

Figure 4 shows the average blocking probability for 27 Erlangs of traffic after each batch is added for 50,00 calls per batch. As more batches are added, the overall blocking probability approaches the final blocking probability of 0.0202 with $99 \%$ confidence interval. The simulation was continued for approximately 19 batches until $99 \%$ confidence interval in results was achieved. In other words, if a long simulation was performed with one million call arrivals, it will produce the results with an approximately $99 \%$ confidence. 


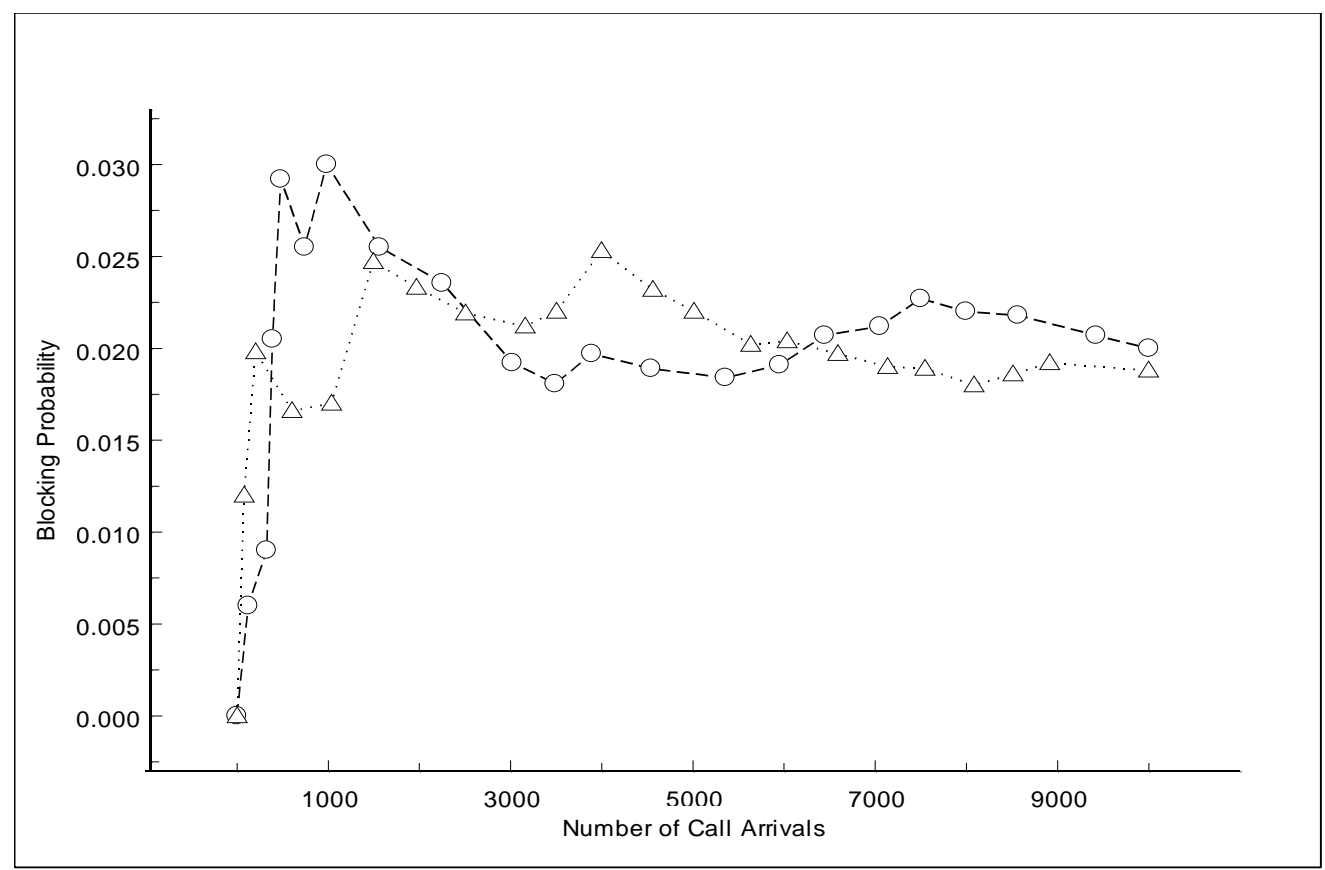

Figure 2: Blocking probability versus call arrivals for the first 10,000 call arrivals for two different simulation runs with different random numbers.

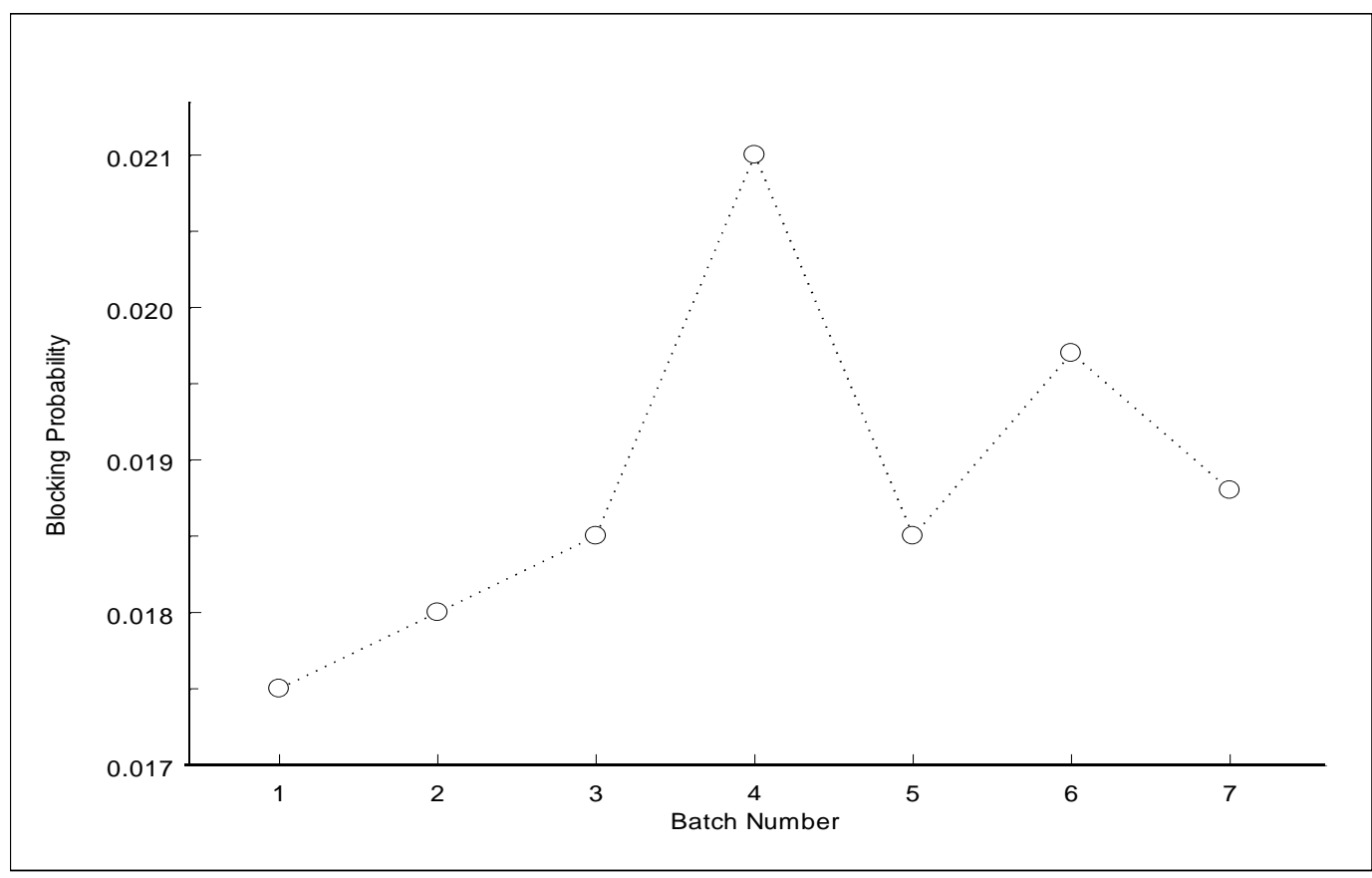

Figure 3: Blocking probability versus batch number for the first seven batches each with 5,000 call arrivals. 


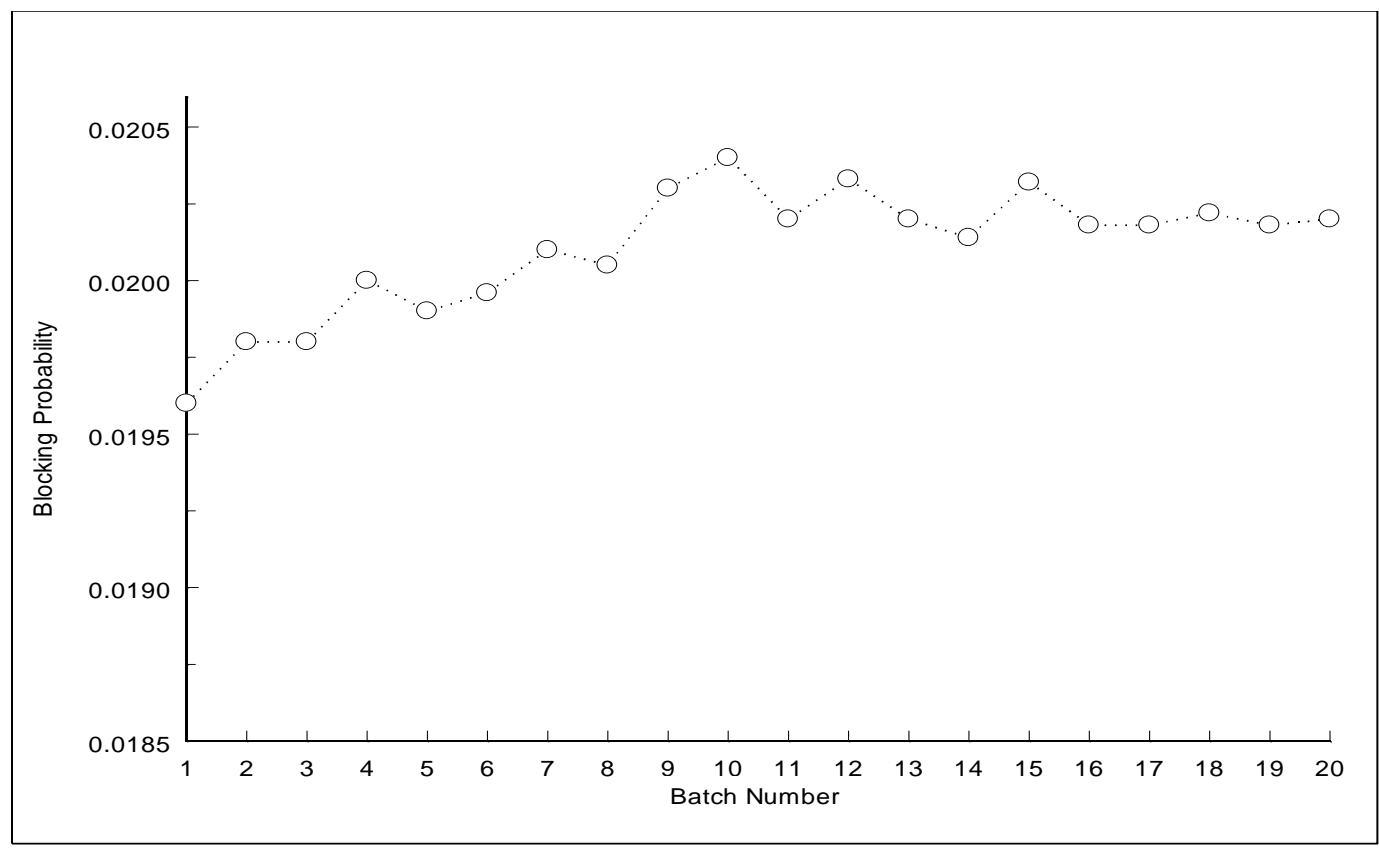

Figure 4: Average blocking probability versus batch number each with 50,000 call arrivals.

\section{Conclusions}

Discrete event simulation method by batch-means method of $\mathrm{M} / \mathrm{M} / \infty$ queuing system was used in simulation of CDMA system. Simulation with 5,000 and 50,000 call arrivals per batch, was used to end the simulation when $95 \%$ and $99 \%$ confidence in results was required. For 50,000 calls per batch, 19 batches were requited to reach $99 \%$ confidence interval and provide the average blocking probability of 0.0202 for the system under study. In other words, it was found that one long simulation with one million arrivals produce the same or better than 99\% confidence interval in results. Twenty batches of 5000 calls each were required (100,000 calls) to achieve $95 \%$ precision in the results providing probability of blockage of 0.0192. The blocking probability per batch randomness substantially decreased as the number of calls per batch increased from 5,000 to 50,000. As the number of batches increased the total average blocking probability approached a steady-state value.

The impact of warm-up period was also analysed by determining the blocking probability against the number of call arrivals. Initially the blocking probability was zero but it gradually increased as more calls were blocked. The simulation results of the first 700 calls for three simulations determined that the blocking probability was so random for each initial stage of the simulation (warm-up period). For the three simulations runs, the blocked calls were call numbers $(62,98,106,113, \ldots)$ and $(79,88,98,202, \ldots)$ and $(124,318,327,379, \ldots)$ respectively. Due to randomness of the CDMA parameters, one could not estimate which calls would be lost and therefore the average blocking probability fluctuated rapidly during the first 700 calls finally approaching 0.015-0.023 range. The blocking probability range was zero to 0.037 for the first 700 calls. When the number of calls arrivals increased to 10,000 and simulation was performed for two simulation runs, the randomness in results decreased approaching the blocking probability range of $0.018-0.020$.

\section{References}

[1] W. H. Tranter and K. L. Kosbar, "Simulation of communication systems," IEEE Communications Magazine, vol. 32, pp. 26-35, 1994.

[2] M. F. Aburdene, Computer Simulation of Dynamic Systems. Dubuque, Iowa: W. C. Brown Publishers, 1988.

[3] K. Pawlikowski, H. D. Jeong, and J. S. Lee, "On credibility of simulation studies of telecommunication networks," IEEE Communications Magazine, vol. 40, pp. 132-9, 2002. 
[4] M. Tanner, Practical Queuing Analysis. Berkshire, England: McGraw-Hill Book Company, 1995.

[5] S. S. Kolahi, "Impact of propagation parameters on neighbouring cells interference in power controlled CDMA systems," IEEE Tencon '05, Melbourne, 2005.

[6] S. S. Kolahi, A. G. Williamson, and K. W. Sowerby, "Other-cell interference in CDMA systems," Electronics Letters, vol. 40, pp. 1134-1135, 2004.

[7] J. S. Evans and D. Everitt, "On the teletraffic capacity of CDMA cellular networks," IEEE Transactions on Vehicular Technology, vol. 48, pp. 153-165, 1999.

[8] J. Evans and D. Everitt, "Analysis of reverse link traffic capacity for cellular mobile communication networks employing Code Division Multiple Access," Australian Telecommunication Networks and Applications Conference, pp. 775-780, 1994.

[9] D. D. Lee, D. H. Kim, Y. J. Chung, H. G. Kim, and K. C. Whang, "Other cell interference with power control in macro/microcell CDMA networks," IEEE Vehicular Technology Conference, vol. 2, pp. 1120-1124, 1996.

[10] S. S. Kolahi, A. G. Williamson, and K. W. Sowerby, "Sensitivity analysis of the reverse link Erlang capacity of CDMA systems," 2004 IEEE Wireless Communications and Networking Conference, WCNC'04, pp. 2220-2225, 2004.

[11] S. S. Kolahi, "On Erlang capacity of CDMA systems," in Mobile and Wireless Communications, C. G. Omidyar, Ed. Norwell, MA: Kluwer Academic Publishers, pp. 227-232, 2003.

[12] K. Pawlikowski, "Steady state simulation of queueing processes: survey of problems and solutions," $A C M$ Computing Surveys, pp. 123-170, 1990.

[13] G. S. Fishman, "Grouping Observations in Digital Simulation," Management Science, pp. 510-521, 1978.

[14] P. T. Strait, Probability and Statistics with Applications. New York: Harcourt Brace Jovanovich inc, 1983.

[15] G. Bancroft and G. O'sullivan, Maths and Statistics. Berkshire, UK: McGraw Hill Book Company, 1998.

[16] R. Barrett, Mathematics with Statistics. Auckland: ESA Publications New Zealand, 1996.

[17] G.N. Higginbottom, Performance Evaluation of Communication Networks. Boston and London: Artech House, 1998.
[18] L. Kleinrock and R. Gaid, Queueing Systems, Problems and Solutions. Los Angles, USA: John Wiley and Sons Inc, 1996.

[19] L. Kleinrock, Queueing Systems, Volume II: Computer Applications. New York and London: John Wiley \& Sons, 1975.

[20] J. F. Hayes and T. Babu, Modeling and Analysis of Telecommunications Networks. Hoboken, NJ: John Wiley \& Sons, 2004.

[21] L. Kleinrock, Queueing Systems, Volume I: Theory. New York and London: John Wiley \& Sons, 1975.

[22] A. M. Viterbi and A. J. Viterbi, "Erlang capacity of a power controlled CDMA system," IEEE Journal on Selected Areas in Communications, vol. 11, pp. 892-900, 1993. 